\title{
Intra-coronary Imaging for the Evaluation of Plaque Modifications Induced by Drug Therapies for Secondary Prevention
}

\author{
Ismail Dogu Kilic ${ }^{1}$ - Enrico Fabris ${ }^{2}$ (D) - Elvin Kedhi ${ }^{3}$. Liviu-Nicolae Ghilencea ${ }^{4}$ - Gianluca Caiazzo ${ }^{5}$. Sara Abou Sherif ${ }^{6}$ \\ Carlo Di Mario ${ }^{7}$
}

Accepted: 16 September 2020 / Published online: 6 October 2020

(C) The Author(s) 2020

\begin{abstract}
Purpose of Review Patients diagnosed with coronary artery disease are at a high risk of subsequent cardiovascular events; therefore, secondary prevention in the form of therapeutic lifestyle changes, and drug therapies is vital. This article aims to review potential application of intra-coronary imaging for the evaluation of plaque modifications, induced by medications for secondary prevention for CAD.

Recent Findings Intra-coronary imaging provides detailed information on the atherosclerotic plaque which is the primary pathological substrate for the recurrent ischemic cardiovascular events. These modalities can detect features associated with high risk and allow serial in vivo imaging of lesions. Therefore, intravascular imaging tools have been used in landmark studies and played a role in improving our understanding of the disease processes.

Summary Changes in size and plaque composition over time can be evaluated by these tools and may help understanding the impact of a treatment. Moreover, surrogate imaging end points can be used when testing new drugs for secondary prevention.
\end{abstract}

Keywords Coronary artery disease $\cdot$ Coronary atherosclerosis $\cdot$ Vulnerable plaque $\cdot$ Intra-coronary imaging $\cdot$ Secondary prevention

This article is part of the Topical Collection on Coronary Heart Disease

Enrico Fabris

enrico.fabris@hotmail.it

Carlo Di Mario

carlo.dimario@unifi.it

1 Department of Cardiology, Pamukkale University Hospitals, Denizli, Turkey

2 Cardiovascular Department, University of Trieste, Trieste, Italy

3 Department of Cardiology, Isala Heart Center, Zwolle, the Netherlands

4 University of Medicine and Pharmacy Carol Davila, Bucharest, Romania

5 P.O. San Giuseppe Moscati, Aversa, Italy

6 Brighton and Sussex Medical School, Brighton, UK

7 Cardio-toraco-vascular Department, Careggi University Hospital, Florence, Italy

\section{Introduction}

Patients diagnosed with coronary artery disease (CAD) are at a high risk of subsequent cardiovascular events. Modifications of risk factors can significantly reduce these recurrent events and premature death amongst these patients. For that reason, secondary prevention in the form of therapeutic lifestyle changes, and drug therapies is vital. The evaluation of treatment effect and risk stratification during follow-up is important because this may enable more intensive treatment, motivation and strict follow-up to reduce clinical outcomes. In addition to clinical outcomes, a number of tools are used to evaluate the impact of preventive strategies including biomarkers or imaging modalities. With regard to imaging of coronary atherosclerosis, intravascular modalities are able to provide high-resolution images that neither angiography nor other non-invasive modalities could. These techniques have been used in landmark studies and played a role in improving the understanding of the disease processes and the prevention strategies. Therefore, this article aims to review potential application of intra-coronary imaging (ICI) for the evaluation of plaque modifications induced by medications for secondary prevention for CAD. 
Why Is Intra-coronary Imaging Important?

\section{ICI for Better Definition of Atherosclerotic Coronary Disease}

Atherosclerotic plaque is the primary pathological substrate leading to ischemic cardiovascular events, and interventions that have favourable effects on the atherosclerotic plaque are expected to improve clinical outcomes [1]. Therefore, atherosclerosis imaging can be utilized as an indicator of the ongoing pathologic process and response to therapeutic interventions. Coronary angiography has been the gold standard for detecting and guiding the treatment of atherosclerotic coronary artery disease. However, despite objective and reproducible measurements can be obtained by quantitative coronary angiography (QCA), it remains limited to merely reflecting the degree of lumen intrusion of atherosclerotic lesion in the contrast filled lumen. Especially in the early stages of the disease, plaques can show an outward growth, known as positive remodelling, without luminal compromise, not detectable by conventional angiography. Furthermore, the characteristics and composition of the plaque cannot be adequately studied by angiography, which only shows large calcifications and ulcerations. Finally various factors such as diffuse disease, vessel foreshortening, angulation, overlap, calcification, eccentricity and contrast streaming make angiographic assessment challenging [2]. Therefore, ICI modalities have been developed to overcome these limitations of conventional angiography. Moreover, ICI provides detailed evaluation of the plaques to discriminate between high- and low-risk lesions and/or patients. From pathological and clinical studies, we know that plaques at high risk for rupture share certain characteristics [3]. Various ICI modalities can detect these features associated with high risk and allow serial in vivo imaging of lesions, contributing to our understanding of the natural history of plaques and the effect of treatment. In fact, detecting high-risk features potentially helps in identifying patients in need of intensive anti-atherosclerotic regimens. The opposite is also true for the lower risk groups. If we can truly classify these patients, intensity of the treatments can be softened and decrease drug-related side effects, and repeated follow-up visits can be safely avoided allowing us to concentrate on truly high-risk individuals. Consequently, if we manage to focus our efforts in the group at higher risk, we may increase their compliance with regular follow-up visits and predict new acute events with frequent provocative tests [4]. Finally, atherosclerosis is a systemic disease; thus, focal therapies are by no means the main therapy. However, in the presence of an effective focal treatment strategy, to help reduce the number of patients with high-risk plaques, ICI may contribute by helping to identify a certain subset of patients who may benefit from these therapies, alongside systemic treatments [4].
ICI for Evaluation of Treatment Plaque Modification

ICI provides a close look to the atherosclerotic plaque, which cannot be substituted by any non-invasive imaging. Consequently, changes in size and composition over time can be evaluated in matched arterial segments. These changes not only help understanding the impact of a treatment but can also be used as a surrogate clinical end point, which is important in testing new drugs [5]. Secondary prevention by means of vigorous risk modification and consistent use of antiplatelet and lipid-lowering agents according to guidelines significantly reduces event rates also in the placebo arm of trials testing new drugs, requiring studies on a larger numbers of patients over longer periods of time in order to reach a sufficient event rate to provide a sufficient statistical power and making the demonstration of an incremental effect of new agents more challenging [6]. On the other hand, surrogate imaging end points may allow a smaller group of patients to be studied for a shorter period of time and accelerate the drug development and analysis. This approach may indeed reduce the costs greatly and potentially prevent the risk of advancing inefficient therapies before further testing [7-9].

\section{Treatment and Plaque Modifications}

\section{Statins}

The clinical benefits of statins have consistently been demonstrated in numerous large-scale studies. They are regarded as one of the key drugs in the primary and secondary prevention of CAD [10-12]. Early statin trials employed conventional angiography to assess their effect on the atherosclerotic plaque, in an attempt to demonstrate that aggressive lipidlowering therapy could halt the progression and even promote regression of coronary atherosclerosis [13-15]. However, results of early studies of lipid lowering showed a phenomenon that was later called "the angiographic paradox". The improvements in lumen measurements were unexpectedly small and inconsistent when compared with the large reductions in the clinical event rates [16]. The awareness of the phenomenon of positive remodelling and the importance of plaque characteristic and composition explains why angiography should be replaced by ICI to address directly the plaque changes.

Several small studies with ICI demonstrated favourable effects of statins. In one of the earliest studies utilizing IVUS, Takagi et al. showed that pravastatin reduced progression of coronary artery atherosclerotic plaque [17]. An angioscopic study also demonstrated that lipid-lowering therapy by statins dramatically decreased the yellow grade of coronary plaque [18]. Benefits of statins, particularly highintensity statin therapy, have been shown in further larger 
studies with ICI. The REVERSAL study comparing pravastatin $40 \mathrm{mg}$ vs atorvastatin $80 \mathrm{mg}$ for 18 months revealed a significant increase in IVUS-determined atheroma volume in pravastatin arm whereas no significant change in intensive therapy arm [19••]. Subsequently, the ASTEROID study showed, for the first time, that very intensive cholesterol lowering with rosuvastatin can even regress the atheroma burden ( $0.79 \%$ reduction in atheroma volume detected by IVUS) $[20 \bullet$. This effect of intensive statin therapy was further explored in the SATURN. Despite the lower level of LDL-C and the higher level of HDL-C achieved with maximal doses of rosuvastatin compared with atorvastatin, both treatment arms resulted in significant but comparable regression of coronary atherosclerosis during 104 weeks of therapy [21••]. Around about two-thirds of study patients demonstrated a regression of atherosclerosis and a reduction in the primary efficacy end point, percent atheroma volume in atorvastatin and rosuvastatin groups found to be $0.99 \%$ and $1.22 \%$, respectively. Both this frequency and level of regression were exceptional, as compared with the results of prior IVUS studies $[21 \bullet \bullet]$.

Nonetheless, the fact that plaque regression observed in these studies are only modest (measuring approximately in $1 \%$ level) raises the question of how statin therapy is associated with a significant decrease in cardiovascular events with such small reductions in plaque burden [1].

A possible explanation could be the potential favourable changes in plaque composition. The effect of statins on plaque composition has been firstly investigated using IB-IVUS system by Kawasaki et al. and showed significant reduction in the lipid component [22]. In the IBIS-4 study, patients with STEMI were treated with high-intensity rosuvastatin $(40 \mathrm{mg} /$ day) over a 13-month period. Despite significant atheroma regression, no change in VH-IVUS defined percent necrotic core was observed during serial examinations [23•]. Likewise, in the radiofrequency-IVUS subset of SATURN including 71 patients, a change in necrotic core volume did not accompany regression of coronary atheroma with maximally intensive statin therapy [24].

Compositional changes with statins have also been investigated using near infrared spectroscopy (NIRS). NIRS can detect and quantify the presence of lipid core in the atherosclerotic plaque (Fig. 1), and NIRS-IVUS catheters may associate it with other features such as lumen size and plaque architecture [25]. Furthermore, NIRS imaging of nonobstructive coronary territories can aid in identifying patients and segments at higher risk for future events [26].

The YELLOW trial randomized patients with multi-vessel CAD randomized to a treatment of either rosuvastatin $40 \mathrm{mg}$ daily or the standard-of-care lipid-lowering therapy. Even after 6-8 weeks of a short-term intensive statin therapy, a significant reduction in the plaque lipid composition was demonstrated with maximum lipid core burden index in $4 \mathrm{~mm}$
(maxLCBI ${ }_{4 \mathrm{~mm}}$ ) [27•] (see Fig. 2 for example illustrating IVUS and NIRS of a patient in the intensive group). In contrast, IBIS-3 study failed to demonstrate a significant reduction of necrotic core volume or LCBI under high-intensity rosuvastatin therapy for 1 year [28].

Another important change in plaque composition is the calcification of the atherosclerotic plaque. In a recently published study, Puri et al. using IVUS data from a post hoc patient-level analysis of 8 prospective randomized trials on serial changes in coronary atheroma showed that statins promote calcification of the atheroma independently of their plaque-regressive effects [29]. Authors concluded that such results highlight the possible procalcific effects of statins, which may be related to possible plaque-stabilizing effects. Along with these findings, high-resolution OCT images introduced new possibilities into how statins act to stabilize plaques (Fig. 3).

In a study on 40 patients with previous myocardial infarction revealed that the degree of increase of fibrous cap thickness (FCT) was significantly greater in the statin treatment group than in the control group [30]. Recently, investigators showed that atorvastatin therapy at $20 \mathrm{mg}$ /day provided a greater increase in FCT compared with $5 \mathrm{mg}$, which was associated with the decrease in serum atherogenic lipoproteins and inflammatory biomarkers [31]. In the YELLOW II study, which included 85 stable coronary artery patients, subjects were given $40 \mathrm{mg}$ of rosuvastatin every day for 8-12 weeks. Baseline OCT minimal FCT was $100.9 \pm 41.7 \mu \mathrm{m}$ and significantly increased upon follow-up-FCT $(108.6 \pm 39.6 \mu \mathrm{m})$. The changes in FCT were also independently associated with the increase in cholesterol efflux capacity [32•].

\section{PCSK9 Inhibitors}

Proprotein convertase subtilisin/kexin type 9 serine protease (PCSK9) plays a critical role in cholesterol metabolism. By binding to the LDL receptor and promoting their degradation and therefore reducing LDL uptake on the hepatocytes, PCSK9 increases LDL-C levels [33]. Moreover, PCSK9 might contribute to atherosclerosis by mechanisms independent of the LDL-C levels [34]. In the ATHEROREMO-IVUS study, serum PCSK9 levels were linearly associated with the fraction and amount of IVUS-VH necrotic core tissue [35]. Consequently, monoclonal antibodies against PCSK9 have emerged as a new and potent class of cholesterol lowering drugs. Recently, the GLAGOV trial evaluated the effects of the evolocumab on atherosclerosis with IVUS. Nine hundred sixty-eight patients with angiographic coronary disease were randomized to receive either evolocumab or placebo along with the statins for 18 months. Lower levels of cholesterol in the evolocumab group ( $36.6 \mathrm{vs} 93.0 \mathrm{mg} / \mathrm{dL}$ ) was associated with reduction in percent atheroma volume for evolocumab $(-0.95 \%$ vs $+0.05 \%$ in the placebo group) $[36 \bullet \bullet$. 


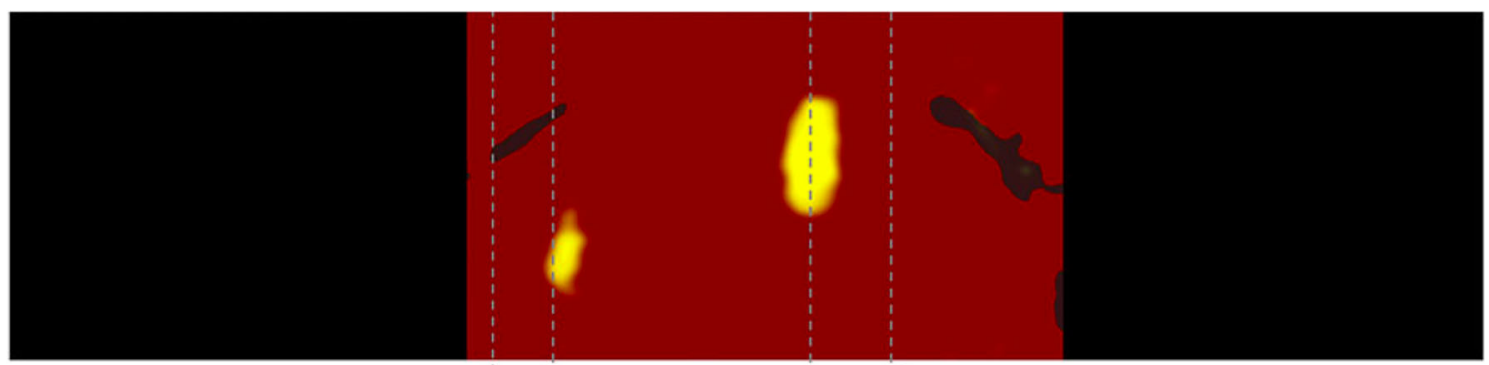

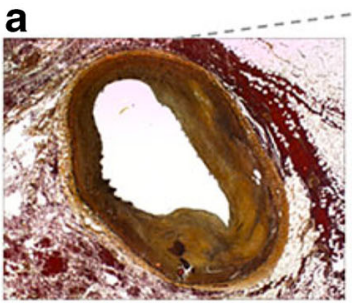

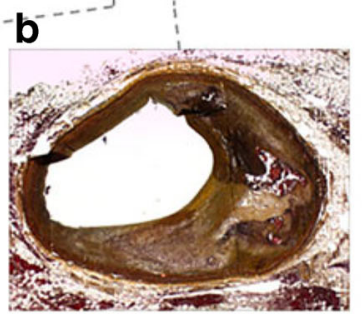

Fig. 1 Example of correlation between NIRS chemogram and histologic findings. Vessel tissue lacking necrotic lipid core corresponds to 'red' in chemogram (a and d-fibrous PIT), whereas necrotic lipid core plaques correspond to 'yellow' (b and c-early stage fibroatheromas). Movat's

Currently ongoing PACMAN-AMI (NCT03067844) study will investigate the effect of the PCSK9 inhibitor alirocumab in a total of 220 acute MI patients undergoing PCI in the
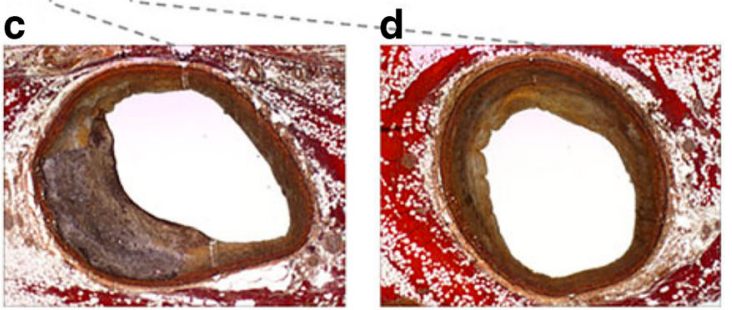

pentachrome stain used for histologic evaluation. (Adapted from: Kilic ID et al. Eur Heart J Cardiovasc Imaging. 2015;16(12):1299-306, by permission of Oxford University Press) [25]

infarct-related artery and receiving guideline-recommended high-intensity statin therapy. A serial, multi-vessel imaging study will be performed to determine the change in plaque
Fig. 2 Top panel: On the left, the baseline angiographic image with FRR evaluation and the intravascular ultrasound crosssectional images of the plaque are shown. On the right, the measurement of lesion lipid core burden index (LCBI) and LCBI at the 4-mm maximal segment $\left(\mathrm{LCBI}_{4 \mathrm{~mm}} \max \right)$ are shown. Bottom panel: On the left, the follow-up angiographic image with FRR re-evaluation and follow-up intravascular ultrasound cross-sectional image are shown. On the right, the reduction of lesion LCBI and $\mathrm{LCBI}_{4 \mathrm{~mm}}$ max are shown. (Adapted from: Kini AS et al. J Am Coll Cardiol. 2013 Jul;62(1):21-9, with permission from Elsevier) [27] a
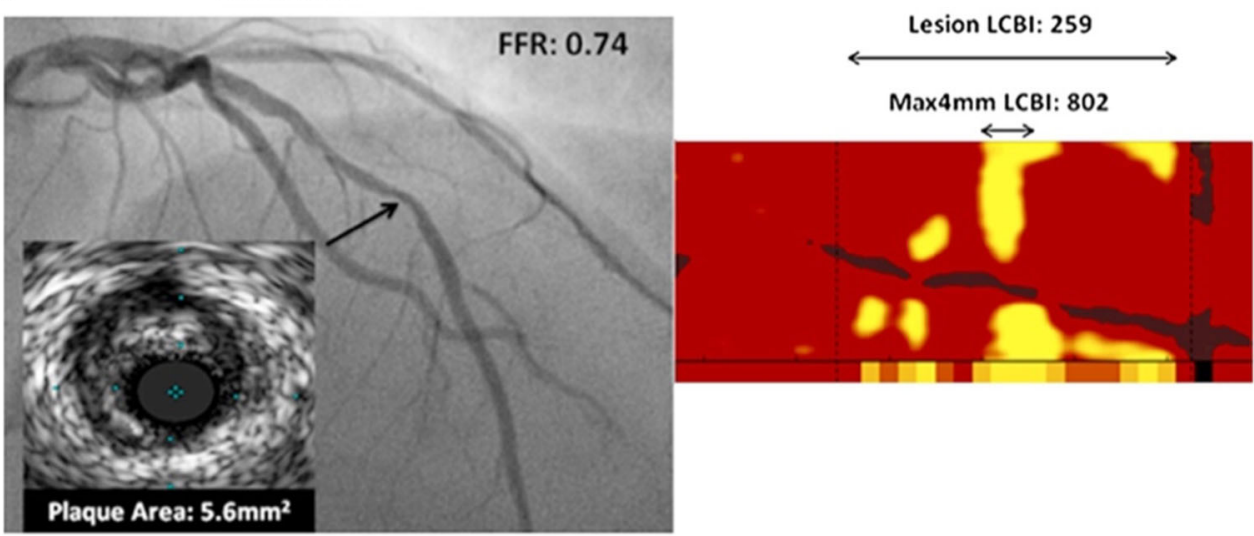

b Follow-up

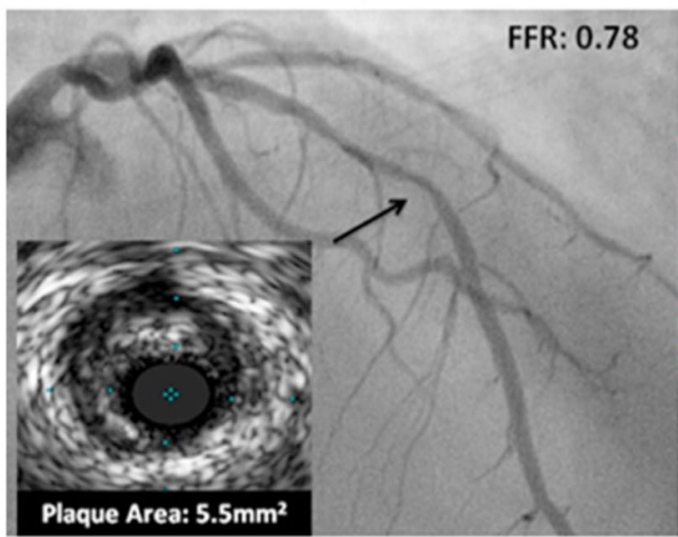

Lesion LCBI: 177

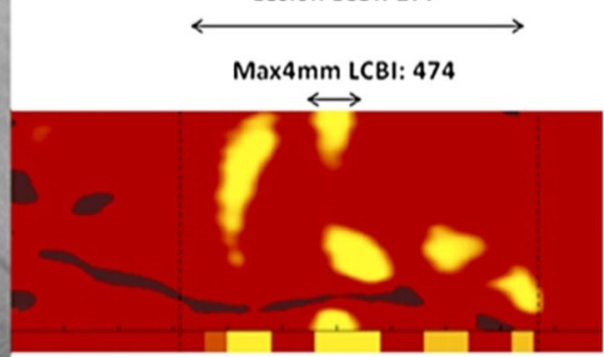


baseline
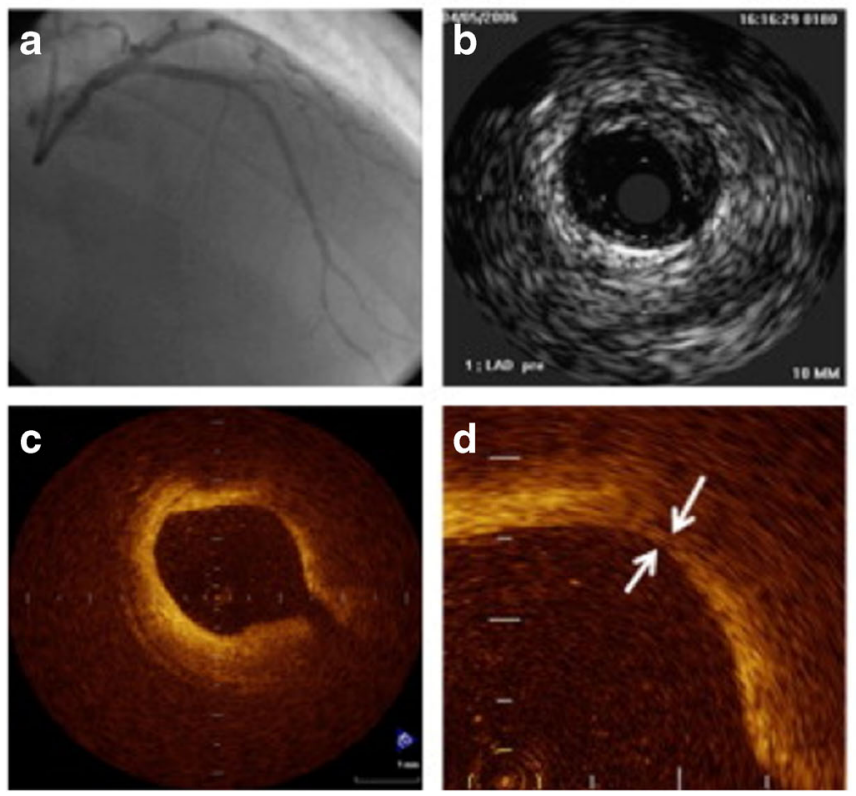

Fig. 3 Coronary and intracoronary images of 47-year-old male patient in the statin treatment group. Four panels illustrate corresponding images with similar angle of coronary angiography (a and $\mathbf{e}$ ) and corresponding cross-sectional images of IVUS (b and f), and OCT (c, $\mathbf{d}, \mathbf{g}, \mathbf{h})$ at baseline and at 9-month follow-up. Fibrous cap thickness at the same cross-

volume, lipid parameters by NIRS, and macrophages and FCT by OCT at week 52 .

\section{Raising HDL}

Several epidemiologic studies provided robust evidence that low levels of high-density lipoprotein cholesterol (HDL-C) are a significant predictor of CV risk [37, 38]. Greater increases in HDL-C are associated with a significantly reduced atheroma progression and lower events even in patients using statins [39]. A pilot study in patients with acute coronary syndromes investigated the effect of recombinant apolipoprotein (apo) A-1 Milano [40], which rapidly mobilized cholesterol and thereby reduced atherosclerotic plaque burden in experimental atherosclerosis models [41]. In this study, the recombinant ApoA-I Milano/phospholipid complex (ETC216) administered intravenously for 5 doses at weekly intervals resulted significant regression of coronary atherosclerosis as measured by IVUS [40]. Further analysis from this study showed that regression of atherosclerotic plaque was characterized by a concomitant shrinkage of the EEM, which results in a lumen size that is virtually unchanged [42]. In other words, large changes in atheroma volume can occur with only minor luminal changes, which can be undetectable by angiography, supporting the importance of ICI.

Another attractive strategy for raising HDL levels is via inhibiting cholesteryl ester transfer protein (CETP). Torcetrapib was the first CETP inhibitor evaluated in phase follow-up in 9 month
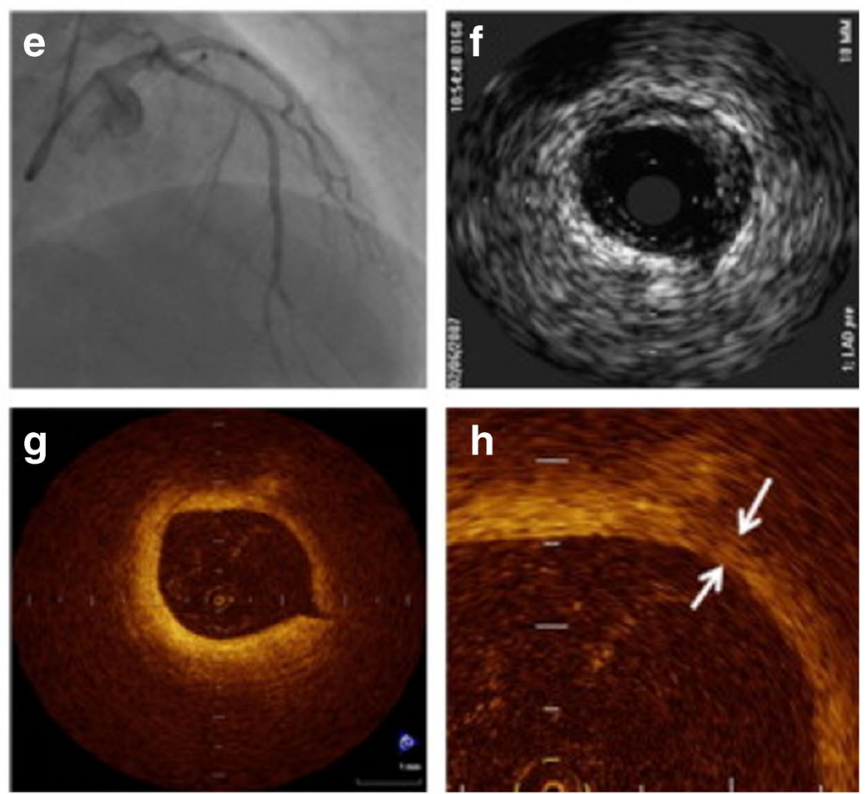

sectional image was increased from 110 (white arrows in D) to $320 \mathrm{~m}$ (white arrows in $\mathrm{H}$ ) during 9-month follow-up period assessed by OCT. (Adapted from: Takarada S et al. Atherosclerosis. 2009 Feb; 202(2):4917, with permission from Elsevier) [30]

III clinical trials; however, despite a substantial increase in HDL-C and decrease in LDL-C, there was no significant decrease in the progression of coronary atherosclerosis when compared with atorvastatin monotherapy in the ILLUSTRATE trial [43]. Moreover, torcetrapib was discontinued after a large clinical study was terminated prematurely due to increased risk of mortality and morbidity rates [44]. However, in a post hoc analysis of ILLUSTRATE trial, the extent of HDL-C increase was found to be inversely related to torcetrapib treatment as well as the degree of regression of atherosclerosis measured by IVUS [45]. Moreover, regression of atherosclerosis was observed in patients who achieved the highest levels of HDL-C with torcetrapib, supporting the notion that the achievement of very high levels of HDL-C via CETP inhibition has the potential to generate functional HDL particles that participate in reverse cholesterol transport [45].

In a recently published study, CER-001 infusions, an engineered lipoprotein particle mimicking pre-beta HDL and consisting of a combination of recombinant human apolipoprotein A-I and two phospholipids, also failed to reduce coronary atherosclerosis on IVUS and QCA when compared with placebo [46]. Finally, in the ApoA-I Synthesis Stimulation and Intravascular Ultrasound for Coronary Atheroma Regression Evaluation (ASSURE) trial, patients with coronary artery disease will be treated over a period of 26 weeks, and change in percentage of atheroma volume will be assessed by IVUS compared with baseline (NCT01067820). 


\section{Lipid Apheresis}

LDL-apheresis is an extracorporeal procedure to remove LDL-C and lipoprotein particles and provides a robust improvement in plasma lipoprotein profiles. In a small study in patients with familial hypercholesterolemia, lipid apheresis in addition to medical therapy resulted in a significant increase in minimal lumen diameter by coronary angiogram and decrease in plaque area by IVUS at 1 year compared with medical therapy alone, suggesting a possible regression of atherosclerosis by LDL-apheresis [47].

\section{Other Systemic Therapies}

Impact of other currently available, such as ezetimibe [48, 49] or experimental non-statin modifying drugs, such as acylcoenzyme A:cholesterol acyltransferase (ACAT), has also been investigated with IVUS. The enzyme esterifies cholesterol in a variety of tissues, and inhibition of the ACAT may prevent excess accumulation of cholesteryl esters in macrophages. However, 2 studies failed to show that ACAT inhibition favourably alters coronary atherosclerosis as assessed by IVUS [50, 51].

Modification of risk factors other than lipid profile also has an impact on the atheroma progression. In PERISCOPE trial, pioglitazone treatment resulted in a significantly lower rate of progression of coronary atherosclerosis compared with glimepiride in patients with type 2 diabetes (DM) and coronary artery disease [52]. Moreover, in patients with DM, once atherosclerosis is established, this is associated with an increased extent, complexity, and a more rapid progression than seen in non-DM patients. In DM patients, certain characteristics beyond ischemia, such as coronary atherosclerosis burden, progression and plaque composition, may need to be considered for a more refined risk stratification in these high-risk patients [53]. Recently, it is reported that DM patients with angiographically intermediate coronary lesions remain at risk for future MACE events, including those patients with FFR negative lesions [54]. Thus, combining functional assessment with imaging techniques may be required to guide our treatment strategy in these patients with high-risk, fastprogressing atherosclerosis. The COMBINE (OCT-FFR) (NCT02989740) is enrolling patients to examine whether the addition of OCT plaque morphological evaluation to FFR hemodynamic assessment of intermediate lesions in DM patients will better predict MACEs and possibly lead to new therapeutic strategies [55].

In the CAMELOT trial which assessed 2 different drug regimens vs placebo in patients with CAD patients, IVUS showed progression in the placebo group, a trend toward progression in the enalapril group, and no progression in the amlodipine group [56]. In the STRADIVARIUS trial, after 18 months of treatment, the weight loss drug rimonabant has failed to show an effect on disease progression for the primary end point percent atheroma volume but showed a favourable effect on total atheroma volume, the secondary end point [57].

The effects of a selective oral inhibitor of lipoproteinassociated phospholipase $\mathrm{A}_{2}$, darapladib, were compared with of placebo in patients with CAD. This enzyme is expressed abundantly in the necrotic core and may contribute plaque vulnerability [58]. After 12 months, there were no significant differences between groups in plaque deformability with IVUS palpography or plasma high-sensitivity $\mathrm{C}$-reactive protein. In the placebo-treated group, however, necrotic core volume increased significantly, whereas darapladib was found to halt this increase, without a significant difference in total atheroma volume [58]. Nonetheless, further clinical trials failed to show a reduction in events in both stable [59] and acute CAD [60].

\section{Local Interventional Therapies}

One of the potential treatment approaches under investigation for the high-risk plaques is the local treatment to sealing by the neo-intima formation which resembles a thick-cap and/or passivating the plaque by local immunomodulation. In this regard, ICI is not capable of detecting these "hot spots", which carry a high risk, but additionally reveals any changes in vulnerable plaque characteristics with these treatments. A small pilot study tested the vShield self-expanding nitinol device (Prescient Medical, Inc., Doylestown, PA, USA), in which high-risk plaques were detected by VH-IVUS and OCT (see Fig. 4). The average baseline FCT, measured by OCT, was 48 $\pm 12 \mu \mathrm{m}$ and increased to $201 \pm 168 \mu \mathrm{m}$ with neo-cap formation in the 6 months follow-up, which might contribute to stability of the plaque [61]. Similarly, using OCT, Brugaletta et al. showed the formation of a neointima layer after the implantation of a bioresorbable vascular scaffold that resembles a thick fibrous cap, which may contribute to stabilization of the plaque [62]. Although the concept is promising, it requires further investigation.

\section{Limitations}

There are also limitations to the utilization of ICI for the studies. To start with, studies using ICI for surrogate end points needs to be interpreted with caution. This is because regardless of how promising a surrogate end point seems, the true clinical benefit may remain uncertain [63]. Also, there is always a possibility for unexpected off-target effects which may counterbalance the favourable effects. As mentioned previously, there are a number of examples where imaging markers showed benefit; however, subsequent clinical trials failed to show a clinical benefit or even showed harm.

Moreover, alterations with therapies do not always signify a clear-cut relation and/or causation. For instance, as 
Fig. 4 a In the upper left, palpogram showing stain value of 1.4\% (Rotterdam Classification III-IV). b In the upper right, corresponding matched thin cap fibroatheroma on IVUS virtual histology analysis with plaque burden of $56 \%$ and necrotic core of $34 \%$ in three consecutive frames. c In the lower left corner, matched OCT frame showing cap thickness of $40 \mu \mathrm{m}$. (Adapted with permission of Europa Group from: Wykrzykowska JJ et al. EuroIntervention. 2012 Dec;8(8):945-54, permission conveyed through Copyright Clearance Center, Inc.) [61]

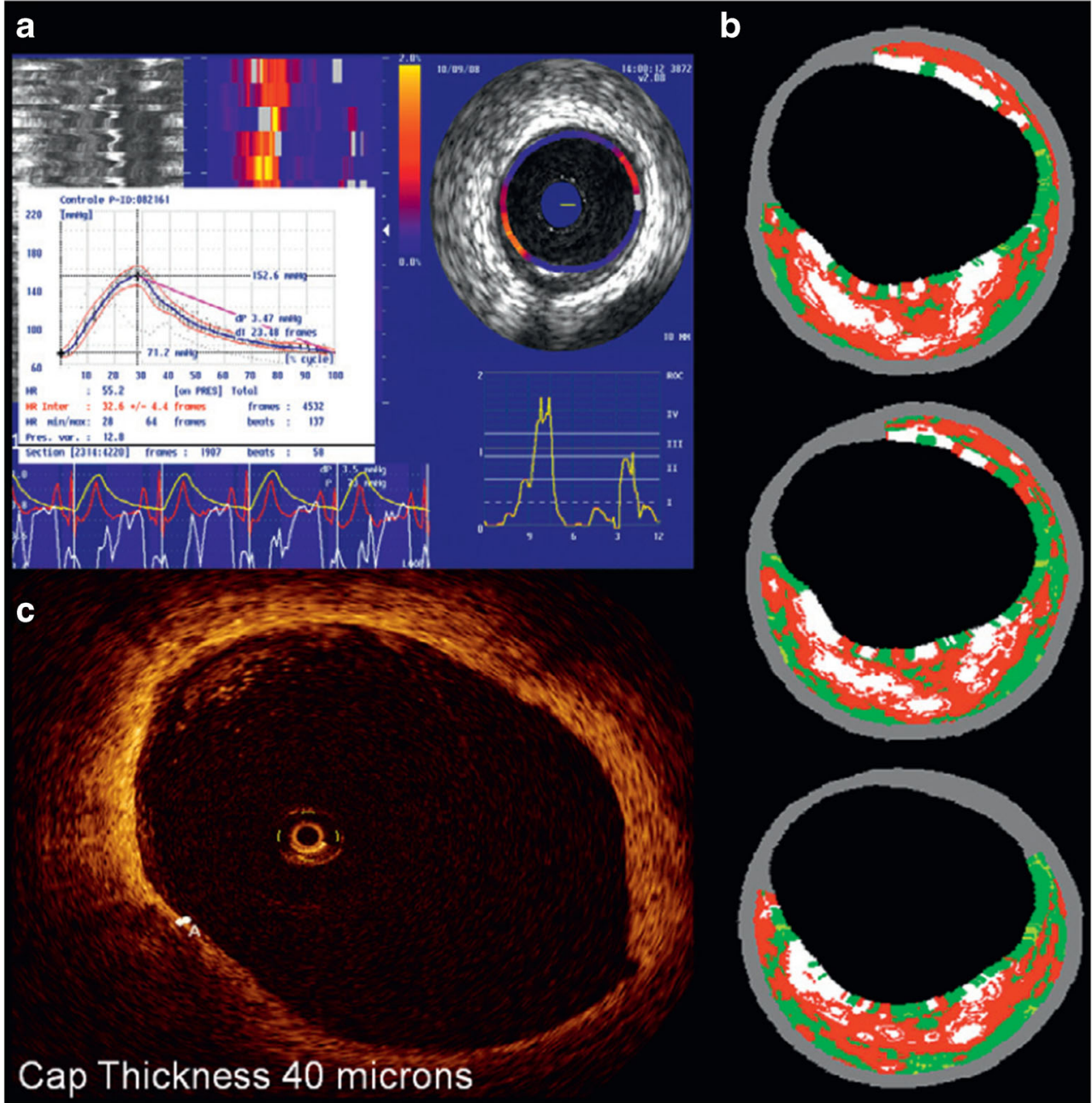

previously mentioned, the reported effects of statins on plaque regression are modest, indicating that despite the statistical significance of this drug therapy, improvements in clinical outcomes may not be directly related to these minute changes in the vessel wall-so the mechanisms of the favourable effects of statins remained speculative.

Furthermore, despite the safety of these modalities are well documented $[64,65]$, they are not completely free of complications, and especially repeated examinations may be of concern with the use of ionizing radiation, contrast media and a need a vascular access.

Moreover, each individual modality provides information on different aspects of the lesion morphology and composition, complementing each other and otherwise lack perfection on their own. Additionally, a major limitation is the inability to assess specific biological processes with the currently available techniques. Molecular imaging techniques are also under development, with an aim to image-specific molecules and cells involved in the pathogenesis of disease using specialized, targeted imaging agents that bind to specific molecular or internalized within a cell [66-68]. In the future, these techniques may potentially allow the functional evaluation of the effect of therapeutic interventions on lesion activity.

There are also technical considerations on the use of ICI. First, acquiring good-quality images is not always possible; moreover, there are a number of artefacts which can preclude accurate measurements [7]. Secondly, matching the arterial segments can sometimes be challenging in different time points, although current co-registration systems may improve the correlations of different techniques. Another limitation is the need for predilatation to cross tight stenoses with the imaging catheter, which can significantly modify the lesion morphology and structure. This also hinders evaluation of totally occluded segments. Similarly, despite it is possible to evaluate the most of the epicardial coronary arteries, catheter-based imaging does not allow the visualization of the entire coronary bed [69].

\section{Conclusions}

ICI provides detailed information on the atherosclerotic plaque, which is the primary pathological substrate for the 
recurrent ischemic cardiovascular events. These modalities have been showed to help investigating the effect of the medications for secondary prevention on the atherosclerotic plaque. Continuous advances in ICI may overcome current limitations and increase utilization of these modalities in the trials or even in risk stratification.

Funding Open access funding provided by Università degli Studi di Trieste within the CRUI-CARE Agreement.

\section{Compliance with Ethical Standards}

Conflict of Interest Dr. Di Mario reports grants from Infraredx, Medtronic, Abbott, Daiichi Sanyo and Shockwave outside the submitted work. Dr. Kedhi reports personal fees from Abbott and Medtronic outside the submitted work. All of the other authors have nothing to disclose.

Human and Animal Rights and Informed Consent This article does not contain any studies with human or animal subjects performed by any of the authors.

Open Access This article is licensed under a Creative Commons Attribution 4.0 International License, which permits use, sharing, adaptation, distribution and reproduction in any medium or format, as long as you give appropriate credit to the original author(s) and the source, provide a link to the Creative Commons licence, and indicate if changes were made. The images or other third party material in this article are included in the article's Creative Commons licence, unless indicated otherwise in a credit line to the material. If material is not included in the article's Creative Commons licence and your intended use is not permitted by statutory regulation or exceeds the permitted use, you will need to obtain permission directly from the copyright holder. To view a copy of this licence, visit http://creativecommons.org/licenses/by/4.0/.

\section{References}

Papers of particular interest, published recently, have been highlighted as:

- Of importance

•. Of major importance

1. Nicholls SJ, Hsu A, Wolski K, Hu B, Bayturan O, Lavoie A, et al. Intravascular ultrasound-derived measures of coronary atherosclerotic plaque burden and clinical outcome. J Am Coll Cardiol. 2010;55(21):2399-407.

2. McDaniel MC, Eshtehardi P, Sawaya FJ, Douglas JS, Samady H. Contemporary clinical applications of coronary intravascular ultrasound. JACC Cardiovasc Interv. 2011;4(11):1155-67.

3. Falk E, Nakano M, Bentzon JF, Finn AV, Virmani R. Update on acute coronary syndromes: the pathologists' view. Eur Heart J. 2013;34(10):719-28.

4. Di Mario C, Moreno PR. Invasive coronary imaging: any role in primary and secondary prevention? Eur Heart J. 2016;37(24): 1883-90.

5. Biomarkers and surrogate endpoints: preferred definitions and conceptual framework. Clin Pharmacol Ther. 2001;69(3):89-95.

6. Nicholls SJ, Sipahi I, Tuzcu EM. Assessment of progression and regression of coronary atherosclerosis by intravascular ultrasound.
A New Paradigm Shift? Rev Española Cardiol (English Ed). 2006;59(1):57-66.

7. Nicholls SJ, Sipahi I, Schoenhagen P, Crowe T, Tuzcu EM, Nissen SE. Application of intravascular ultrasound in anti-atherosclerotic drug development. Nat Rev Drug Discov. 2006;5(6):485-92.

8. Lindsay AC, Choudhury RP. Form to function: current and future roles for atherosclerosis imaging in drug development. Nat Rev Drug Discov. 2008;7(6):517-29.

9. Hartmann M, Huisman J, Böse D, Jensen LO, Schoenhagen P, Mintz GS, et al. Serial intravascular ultrasound assessment of changes in coronary atherosclerotic plaque dimensions and composition: an update. Eur J Echocardiogr. 2011;12(4):313-21.

10. Group SSSS. Randomised trial of cholesterol lowering in 4444 patients with coronary heart disease: the Scandinavian simvastatin survival study (4S). Lancet. 1994;344(8934):1383-9.

11. Shepherd J, Cobbe SM, Ford I, Isles CG, Lorimer AR, MacFarlane $\mathrm{PW}$, et al. Prevention of coronary heart disease with pravastatin in men with hypercholesterolemia. West of Scotland coronary prevention study group. N Engl J Med. 1995;333(20):1301-7.

12. Sacks FM, Pfeffer MA, Moye LA, Rouleau JL, Rutherford JD, Cole TG, et al. The effect of pravastatin on coronary events after myocardial infarction in patients with average cholesterol levels. Cholesterol and recurrent events trial investigators. N Engl J Med. 1996;335(14):1001-9.

13. Blankenhorn DH, Azen SP, Kramsch DM, Mack WJ, CashinHemphill L, Hodis HN, et al. Coronary angiographic changes with lovastatin therapy. The monitored atherosclerosis regression study (MARS). Ann Intern Med. 1993;119(10):969-76.

14. Thompson GR, Hollyer J, Waters DD. Percentage change rather than plasma level of LDL-cholesterol determines therapeutic response in coronary heart disease. Curr Opin Lipidol. 1995;6(6): 386-8.

15. Jukema JW, Bruschke AV, van Boven AJ, Reiber JH, Bal ET, Zwinderman $\mathrm{AH}$, et al. Effects of lipid lowering by pravastatin on progression and regression of coronary artery disease in symptomatic men with normal to moderately elevated serum cholesterol levels. The regression growth evaluation statin study (REGRESS). Circulation. 1995;91(10):2528-40.

16. Brown BG, Zhao XQ, Sacco DE, Albers JJ. Lipid lowering and plaque regression. New insights into prevention of plaque disruption and clinical events in coronary disease. Circulation. 1993;87(6):1781-91.

17. Takagi T, Yoshida K, Akasaka T, Hozumi T, Morioka S, Yoshikawa J. Intravascular ultrasound analysis of reduction in progression of coronary narrowing by treatment with pravastatin. Am J Cardiol. 1997;79(12):1673-6.

18. Takano M, Mizuno K, Yokoyama S, Seimiya K, Ishibashi F, Okamatsu K, et al. Changes in coronary plaque color and morphology by lipid-lowering therapy with atorvastatin: serial evaluation by coronary angioscopy. J Am Coll Cardiol. 2003;42(4):680-6.

19.• Nissen SE, Tuzcu EM, Schoenhagen P, Brown BG, Ganz P, Vogel RA, et al. Effect of intensive compared with moderate lipidlowering therapy on progression of coronary atherosclerosis: a randomized controlled trial. JAMA. 2004;291(9):1071-80 In this landmark study, intensive lipid-lowering treatment with $80 \mathrm{mg}$ atorvastatin reduced progression of coronary atherosclerosis compared with pravastatin in patients with coronary artery disease.

20.• Nissen SE, Nicholls SJ, Sipahi I, Libby P, Raichlen JS, Ballantyne $\mathrm{CM}$, et al. Effect of very high-intensity statin therapy on regression of coronary atherosclerosis: the ASTEROID trial. JAMA. 2006;295(13):1556-65 This study showed, for the first time, that very intensive cholesterol lowering with $\mathbf{4 0} \mathbf{~ m g}$ rosuvastatin can even regress atherosclerosis.

21.• Nicholls SJ, Ballantyne CM, Barter PJ, Chapman MJ, Erbel RM, Libby $P$, et al. Effect of two intensive statin regimens on 
progression of coronary disease. N Engl J Med. 2011;365(22): 2078-87 Maximal doses of rosuvastatin and atorvastatin led to significant and similar degree regression of coronary atherosclerosis, despite the lower level of LDL cholesterol and the higher level of HDL cholesterol with rosuvastatin therapy.

22. Kawasaki M, Sano K, Okubo M, Yokoyama H, Ito Y, Murata I, et al. Volumetric quantitative analysis of tissue characteristics of coronary plaques after statin therapy using three-dimensional integrated backscatter intravascular ultrasound. J Am Coll Cardiol. 2005;45(12):1946-53.

23. Raber L, Taniwaki M, Zaugg S, Kelbaek H, Roffi M, Holmvang L, et al. Effect of high-intensity statin therapy on atherosclerosis in non-infarct-related coronary arteries (IBIS-4): a serial intravascular ultrasonography study. Eur Heart J. 2014;36(8):490-500 Despite significant atheroma regression, no change in VH-IVUS defined per cent necrotic core was observed in patients with STEMI and treated with $40 \mathrm{mg}$ rosuvastatin.

24. Puri R, Libby P, Nissen SE, Wolski K, Ballantyne CM, Barter PJ, et al. Long-term effects of maximally intensive statin therapy on changes in coronary atheroma composition: insights from SATURN. Eur Heart J Cardiovasc Imaging. 2014;15(4):380-8.

25. Kilic ID, Caiazzo G, Fabris E, Serdoz R, Abou-Sherif S, Madden S, et al. Near-infrared spectroscopy-intravascular ultrasound: Scientific basis and clinical applications. Eur Heart J Cardiovasc Imaging. 2015;16(12):1299-306.

26. Waksman R, Di Mario C, Torguson R, Ali ZA, Singh V, Skinner $\mathrm{WH}$, et al. Identification of patients and plaques vulnerable to future coronary events with near-infrared spectroscopy intravascular ultrasound imaging: a prospective, cohort study. Lancet. 2019;394(10209):1629-1637.

27. Kini AS, Baber U, Kovacic JC, Limaye A, Ali ZA, Sweeny J, et al. Changes in plaque lipid content after short-term intensive versus standard statin therapy: the YELLOW trial (reduction in yellow plaque by aggressive lipid-lowering therapy). J Am Coll Cardiol. 2013;62(1):21-9 Even after a short term intensive statin therapy a significant reduction in the plaque lipid composition was demonstrated with NIRS.

28. Oemrawsingh RM, Garcia-Garcia HM, van Geuns RJM, Lenzen MJ, Simsek C, de Boer SPM, et al. Integrated biomarker and imaging study 3 (IBIS-3) to assess the ability of rosuvastatin to decrease necrotic core in coronary arteries. EuroIntervention. 2016;12(6):734-9.

29. Puri R, Nicholls SJ, Shao M, Kataoka Y, Uno K, Kapadia SR, et al. Impact of statins on serial coronary calcification during atheroma progression and regression. J Am Coll Cardiol. 2015;65(13):127382.

30. Takarada S, Imanishi $\mathrm{T}$, Kubo $\mathrm{T}$, Tanimoto $\mathrm{T}$, Kitabata $\mathrm{H}$, Nakamura N, et al. Effect of statin therapy on coronary fibrouscap thickness in patients with acute coronary syndrome: assessment by optical coherence tomography study. Atherosclerosis. 2009;202(2):491-7.

31. Komukai K, Kubo T, Kitabata H, Matsuo Y, Ozaki Y, Takarada S, et al. Effect of atorvastatin therapy on fibrous cap thickness in coronary atherosclerotic plaque as assessed by optical coherence tomography. J Am Coll Cardiol. 2014;64(21):2207-17.

32. Kini AS, Vengrenyuk Y, Yoshimura T, Matsumura M, Pena J, Baber U, et al. Assessment of fibrous cap thickness by optical coherence tomography in vivo: reproducibility and standardization. J Am Coll Cardiol. 2016;69(6):644-57 Baseline Fibrous cap thickness significantly increased upon follow-up with 8-12 weeks of $40 \mathrm{mg}$ rosuvastatin therapy.

33. Bergeron N, Phan BAP, Ding Y, Fong A, Krauss RM. Proprotein Convertase Subtilisin/Kexin Type 9 Inhibition. Circulation. 2015;132(17):1648-66.
34. Urban D, Pöss J, Böhm M, Laufs U. Targeting the proprotein convertase subtilisin/kexin type 9 for the treatment of dyslipidemia and atherosclerosis. J Am Coll Cardiol. 2013;62(16):1401-8.

35. Cheng JM, Oemrawsingh RM, Garcia-Garcia HM, Boersma E, van Geuns R-J, Serruys PW, et al. PCSK9 in relation to coronary plaque inflammation: results of the ATHEROREMO-IVUS study. Atherosclerosis. 2016;248:117-22.

36.• Nicholls SJ, Puri R, Anderson T, Ballantyne CM, Cho L, Kastelein JJP, et al. Effect of evolocumab on progression of coronary disease in statin-treated patients. JAMA. 2016;316(22):2373-2384. Addition of evolocumab to statin therapy resulted in greater LDL - cholesterol lowering and atheroma regression compared to statins alone.

37. Genest JJ, McNamara JR, Salem DN, Schaefer EJ. Prevalence of risk factors in men with premature coronary artery disease. Am J Cardiol. 1991;67(15):1185-9.

38. Assmann G, Schulte H, von Eckardstein A, Huang Y. High-density lipoprotein cholesterol as a predictor of coronary heart disease risk. The PROCAM experience and pathophysiological implications for reverse cholesterol transport. Atherosclerosis. 1996;124(Suppl): S11-20.

39. Puri R, Nissen SE, Shao M, Kataoka Y, Uno K, Kapadia SR, et al. The beneficial effects of raising high-density lipoprotein cholesterol depends upon achieved levels of low-density lipoprotein cholesterol during statin therapy: implications for coronary atheroma progression and cardiovascular events. Eur J Prev Cardiol. 2016;23(5): 474-85.

40. Nissen SE, Tsunoda T, Tuzcu EM, Schoenhagen P, Cooper CJ, Yasin M, et al. Effect of recombinant ApoA-I Milano on coronary atherosclerosis in patients with acute coronary syndromes: a randomized controlled trial. JAMA. 2003;290(17):2292-300.

41. Shah PK, Yano J, Reyes O, Chyu KY, Kaul S, Bisgaier CL, et al. High-dose recombinant apolipoprotein A-I(Milano) mobilizes tissue cholesterol and rapidly reduces plaque lipid and macrophage content in apolipoprotein e-deficient mice. Potential implications for acute plaque stabilization. Circulation. 2001;103(25):3047-50.

42. Nicholls SJ, Tuzcu EM, Sipahi I, Schoenhagen P, Crowe T, Kapadia S, et al. Relationship between atheroma regression and change in lumen size after infusion of apolipoprotein A-I Milano. J Am Coll Cardiol. 2006;47(5):992-7.

43. Nissen SE, Tardif J-C, Nicholls SJ, Revkin JH, Shear CL, Duggan WT, et al. Effect of torcetrapib on the progression of coronary atherosclerosis. N Engl J Med. 2007;356(13):1304-16.

44. Barter PJ, Caulfield M, Eriksson M, Grundy SM, Kastelein JJP, Komajda M, et al. Effects of torcetrapib in patients at high risk for coronary events. N Engl J Med. 2007;357(21):2109-22.

45. Nicholls SJ, Tuzcu EM, Brennan DM, Tardif J-C, Nissen SE. Cholesteryl Ester transfer protein inhibition, high-density lipoprotein raising, and progression of coronary atherosclerosis: insights from ILLUSTRATE investigation of lipid level management using coronary ultrasound to assess reduction of Atherosclerosi. Circulation. 2008;118(24):2506-14.

46. Tardif J-C, Ballantyne CM, Barter P, Dasseux J-L, Fayad ZA, Guertin $\mathrm{M}-\mathrm{C}$, et al. Effects of the high-density lipoprotein mimetic agent CER-001 on coronary atherosclerosis in patients with acute coronary syndromes: a randomized trial $\uparrow$. Eur Heart J. 2014;35(46): 3277-86.

47. Matsuzaki M, Hiramori K, Imaizumi T, Kitabatake A, Hishida H, Nomura M, et al. Intravascular ultrasound evaluation of coronary plaque regression by low density lipoprotein-apheresis in familial hypercholesterolemia: the low density lipoprotein-apheresis coronary morphology and reserve trial (LACMART). J Am Coll Cardiol. 2002;40(2):220-7.

48. Nakajima N, Miyauchi K, Yokoyama T, Ogita M, Miyazaki T, Tamura H, et al. Effect of combination of ezetimibe and a statin 
on coronary plaque regression in patients with acute coronary syndrome. IJC Metab Endocr. 2014;3:8-13.

49. Tsujita K, Sugiyama S, Sumida H, Shimomura H, Yamashita T, Yamanaga K, et al. Plaque REgression with cholesterol absorption inhibitor or synthesis inhibitor evaluated by IntraVascular UltraSound (PRECISE-IVUS trial): study protocol for a randomized controlled trial. J Cardiol 2015

50. Tardif J-C, Grégoire J, L'Allier PL, Anderson TJ, Bertrand O, Reeves F, et al. Effects of the acyl coenzyme a:cholesterol acyltransferase inhibitor avasimibe on human atherosclerotic lesions. Circulation. 2004;110(21):3372-7.

51. Nissen SE, Tuzcu EM, Brewer HB, Sipahi I, Nicholls SJ, Ganz P, et al. Effect of ACAT inhibition on the progression of coronary atherosclerosis. N Engl J Med. 2006;354(12):1253-63.

52. Nissen SE, Nicholls SJ, Wolski K, Nesto R, Kupfer S, Perez A, et al. Comparison of pioglitazone vs glimepiride on progression of coronary atherosclerosis in patients with type 2 diabetes: the PERISCOPE randomized controlled trial. JAMA. 2008;299(13): 1561-73.

53. Kennedy MW, Fabris E, Suryapranata H, Kedhi E. Is ischemia the only factor predicting cardiovascular outcomes in all diabetes mellitus patients? Cardiovasc Diabetol. 2017;16(1):51.

54. Kennedy MW, Hermanides RS, Kaplan E, Hemradj V, Fabris E, Koopmans PC, et al. Fractional flow reserve-guided deferred versus complete revascularization in patients with diabetes mellitus. Am J Cardiol. 2016;118(9):1293-9.

55. Kennedy MW, Fabris E, Ijsselmuiden AJ, Nef H, Reith S, Escaned $\mathrm{J}$, et al. Combined optical coherence tomography morphologic and fractional flow reserve hemodynamic assessment of non- culprit lesions to better predict adverse event outcomes in diabetes mellitus patients: COMBINE (OCT-FFR) prospective study. Rationale and design. Cardiovasc Diabetol. 2016;15(1):144.

56. Nissen SE, Tuzcu EM, Libby P, Thompson PD, Ghali M, Garza D, et al. Effect of antihypertensive agents on cardiovascular events in patients with coronary disease and normal blood pressure: the CAMELOT study: a randomized controlled trial. JAMA. 2004;292(18):2217-25.

57. Nissen SE, Nicholls SJ, Wolski K, Rodés-Cabau J, Cannon CP, Deanfield JE, et al. Effect of rimonabant on progression of atherosclerosis in patients with abdominal obesity and coronary artery disease: the STRADIVARIUS randomized controlled trial. JAMA. 2008;299(13):1547-60.

58. Serruys PW, García-García HM, Buszman P, Erne P, Verheye S, Aschermann M, et al. Effects of the direct lipoprotein-associated phospholipase a(2) inhibitor darapladib on human coronary atherosclerotic plaque. Circulation. 2008;118(11):1172-82.

59. White HD, Held C, Stewart R, Tarka E, Brown R, Davies RY, et al. Darapladib for preventing ischemic events in stable coronary heart disease. N Engl J Med. 2014;370(18):1702-11.

60. O'Donoghue ML, Braunwald E, White HD, Steen DP, Lukas MA, Tarka E, et al. Effect of darapladib on major coronary events after an acute coronary syndrome: the SOLID-TIMI 52 randomized clinical trial. JAMA. 2014;312(10):1006-15.

61. Wykrzykowska JJ, Diletti R, Gutierrez-Chico JL, van Geuns RJ, van der Giessen WJ, Ramcharitar S, et al. Plaque sealing and passivation with a mechanical self-expanding low outward force nitinol vShield device for the treatment of IVUS and OCT-derived thin cap fibroatheromas (TCFAs) in native coronary arteries: report of the pilot study vShield evaluated at. EuroIntervention. 2012;8(8): 945-54.

62. Brugaletta S, Radu MD, Garcia-Garcia HM, Heo JH, Farooq V, Girasis C, et al. Circumferential evaluation of the neointima by optical coherence tomography after ABSORB bioresorbable vascular scaffold implantation: can the scaffold cap the plaque? Atherosclerosis. 2012;221(1):106-12.

63. Aronson JK. Biomarkers and surrogate endpoints. Br J Clin Pharmacol. 2005;59(5):491-4.

64. Batkoff BW, Linker DT. Safety of intracoronary ultrasound: data from a multicenter European registry. Catheter Cardiovasc Diagn. 1996;38(3):238-41.

65. Imola F, Mallus MT, Ramazzotti V, Manzoli A, Pappalardo A, Di Giorgio A, et al. Safety and feasibility of frequency domain optical coherence tomography to guide decision making in percutaneous coronary intervention. EuroIntervention. 2010;6(5):575-81.

66. Osborn EA, Jaffer FA. The advancing clinical impact of molecular imaging in CVD. JACC Cardiovasc Imaging. 2013;6(12):1327-41.

67. Jaffer FA, Verjans JW. Molecular imaging of atherosclerosis: clinical state-of-the-art. Heart. 2014;100(18):1469-77.

68. Vinegoni C, Botnaru I, Aikawa E, Calfon MA, Iwamoto Y, Folco $\mathrm{EJ}$, et al. Indocyanine green enables near-infrared fluorescence imaging of lipid-rich, inflamed atherosclerotic plaques. Sci Transl Med. 2011;3(84):84ra45.

69. Brown BG, Zhao X-Q. Is intravascular ultrasound the gold standard surrogate for clinically relevant atherosclerosis progression? J Am Coll Cardiol. 2007;49(9):933-8.

Publisher's Note Springer Nature remains neutral with regard to jurisdictional claims in published maps and institutional affiliations. 